\title{
Update on Photodynamic Therapy for Age-related Macular Degeneration
}

\author{
a report by \\ Murat Karacorlu, MD, MSc
}

Professor of Ophthalmology and Director, Istanbul Retina Institute DOI: 10.17925/USOR.2007.02.00.25

The leading cause of legal blindness in patients over the age of 60 years in developed nations is age-related macular degeneration (AMD). ${ }^{1,2}$ In the US, the number of people affected by AMD is predicted to increase by over $50 \%$ by the year 2020, and will account for roughly one-sixth of the population. ${ }^{3}$ AMD is broadly classified into two types: non-exudative (dry) and exudative (wet). Although the exudative form is less common, it causes the most damage. The Beaver Dam Eye Study reported that in a population aged $43-86$ years non-exudative AMD occured in $15.6 \%$ and exudative $A M D$ in $1.2 \%$. Of those patients who progress to blindness from $A M D$, the non-exudative form accounts for roughly $15 \%$, while the exudative form is responsible for $85 \% \%^{2,4,5}$ Non-exudative AMD is characterized in its earlier stages by changes at the level of the retinal pigment epithelium (RPE) and Bruch's membrane, including the formation of drusen and RPE pigmentary abnormalities. Later, geographical atrophy (GA) can develop, which also involves atrophy of the choriocapillaris and outer segments of the photoreceptors, along with RPE loss. ${ }^{4}$

GA is defined as an area of RPE cell loss with a diameter greater than $175 \mu \mathrm{m}$. Although termed 'atrophy,' the basis of the lesion is RPE cell loss. GA typically occurs in the parafoveal area first, but may progress to involve the fovea in later disease. ${ }^{6}$ The development of GA is thought to be influenced by the lipofuscin content of the respective RPE cells. The RPE cells in the posterior pole, particularly the parafoveal macula, typically have the greatest concentrations of lipofuscin. ${ }^{7}$ Patients with bilateral GA have roughly a $4 \%$ risk over two years of developing choroidal neovascularization (CNV). ${ }^{8}$ Patients with CNV in the other eye have an $18 \%$ chance over two years of developing CNV in the study eye harboring GA. Among those patients with GA and 20/50 or better visual acuity (VA), half lose three lines and one-quarter lose six lines of vision over the subsequent two years. ${ }^{9}$

Exudative AMD is defined by the growth of new blood vessels, or neovascularization. Typically, neovascularization begins in the choroids, extends through a break in Bruch's membrane, and proliferates. The neovascularization may also traverse the RPE and proliferate in the subretinal space. The new vessels are often incompetent and leak fluid and blood into the subretinal or sub-RPE spaces. Chronic fluid can lead to lipid exudation. Later in the course of exudative $A M D$, there are cicatrizing changes, referred to as disciform scars, with poor accompanying VA, due to scarring and atrophy of the RPE and photoreceptor layers. ${ }^{4}$

Ocular photodynamic therapy (PDT) is one of the latest treatments for CNVs, which are the leaky vascular structures under the retina in the 'wet' form of AMD. PDT utilizes an innovative concept to treat CNVs without damaging overlying or nearby retinal or other ocular tissues. Ocular PDT has been introduced as a novel treatment for several subtypes of neovascular AMD and CNV secondary to pathological myopia..$^{10-12}$ Recent studies indicate that PDT may also be used to treat CNV secondary to various vascular retinochoroidal diseases, such as choroiditis, retinochoroiditis, angioid streaks, central serous chorioretinopathy (CSCR), and parafoveal telangiectasia. Furthermore, it may be used to treat CNV associated with macular dystrophy and idiopathic CNV, as well as diseases without any CNV, such as choroidal and retinal hemangioma, retinal hamartoma, choroidal melanoma, chronic CSCR, angiomatous lesions secondary to systemic diseases, rubeosis iridis, or neovascular glaucoma. ${ }^{12-19}$

\section{Mechanism of Action}

PDT using verteporfin (Visudyne ${ }^{\mathrm{TM}}$, Novartis Ophthalmic, Switzerland) selectively targets vascular endothelial cells. By damaging the intraluminal portion of the exposed vessel, the treatment achieves a selective vascular occlusion without affecting the adjacent neural structures. PDT causes reduced leakage and vessel occlusion by two possible mechanisms. First, the specificity and uptake of verteporfin for target cells with a high expression of low-density lipoprotein (LDL) receptors-such as tumor and neovascular endothelial cells - is enhanced by the use of a liposomal formulation and its rapid uptake by plasma LDL. ${ }^{20-21}$ Second, whereas normal vessels are usually spared phototoxic effect due to their intact blood-retina barrierpreventing any extravasation of the photosensitization within the nonfenestrated vascular wall-pathological leakage in choroidal and retinal hemangiomas and CNV may contribute to the vascular occlusion. ${ }^{22}$

\section{Application}

The main advantage of PDT over conventional laser treatment remains the selective thrombosis of new vessels while preserving adjacent neuroretinal structures. ${ }^{23}$ PDT is a two-step procedure. The photosensitizer verteporfin is

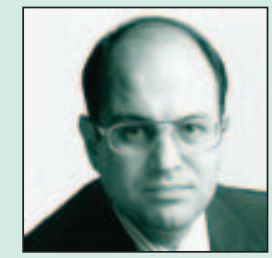

Murat Karacorlu, MD, MSc, is Professor of Ophthalmology and Director of the Istanbul Retina Institute. He has served as a Board Member and Chairman of the Retina Section of the Turkish Society of Ophthalmology. He has also served as a member of the organizing committees of many national and international congresses, and was President of the European Society of Ophthalmology (ESO) 2001 Scientific Committee. He has published extensively in peer-reviewed journals. Professor Karacorlu is Associate Editor of Acta Scandinavica Ophthalmologica and a reviewer for the American Journal of Ophthalmology, the British Journal of Ophthalmology, Ophthalmology, Retina, and Investigative Ophthalmology and Visual Science. His main areas of scientific interest are age-related macular degeneration, retinovascular and hereditary retinal diseases, retinopathy of prematurity, imaging, electrophysiology, and vitreo-retinal surgery.

E: mkaracorlu@superonline.com 
A

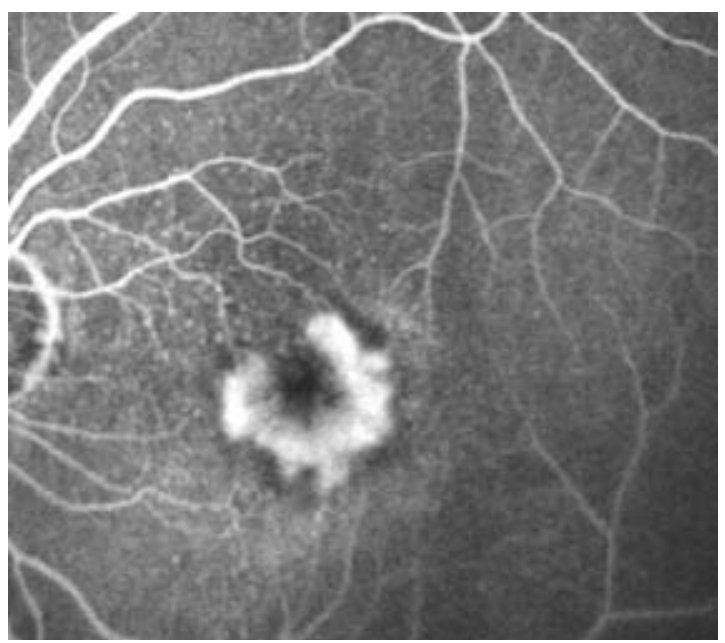

B

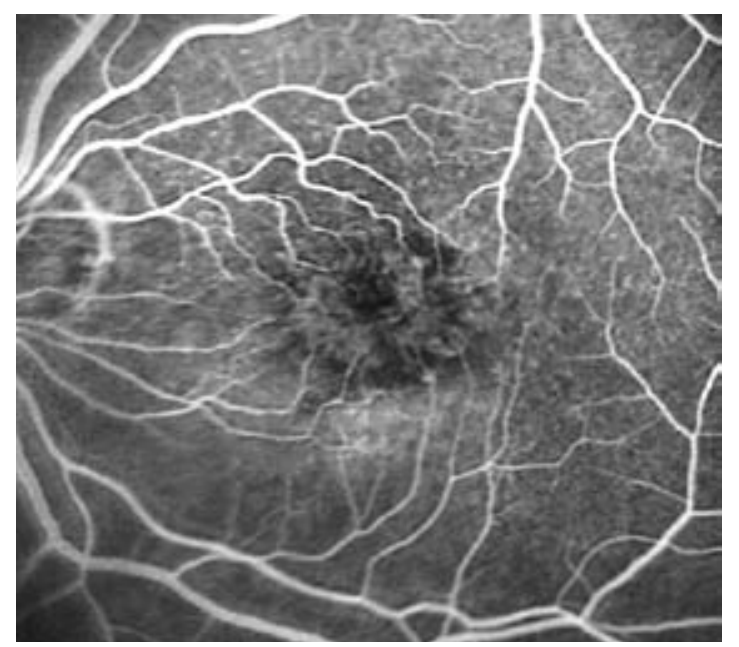

Before (A) and after (B) photodynamic therapy (PDT). The leakage (hyperfluorescence) decreased after the PDT.

first injected intravenously, then the dye in the target tissue is selectively activated using a laser light. The diameter of the treatment spot is calculated based on the lesion size measured on the pre-treatment fluorescein angiogram (FA). To the greatest linear dimension of the retinal lesion, $1,000 \mu \mathrm{m}$ are added. Five minutes after a 10-minute infusion of the photosensitizer (at a dosage of $6 \mathrm{mg} / \mathrm{m}^{2}$ of body surface area), a diode laseremitting light at $689 \mathrm{~nm}$ for photosensitization is used to irradiate the lesion, delivering a light dose of $50 \mathrm{~J} / \mathrm{cm}^{2}$ at an irradiance of $600 \mathrm{~mW} / \mathrm{cm}^{2}$ over $83 \mathrm{~s}$.

\section{What to Expect After Photodynamic Therapy}

Two-year randomized clinical trials with PDT demonstrated its beneficial effect over placebo for classic CNV in AMD and for CNV secondary to myopia. ${ }^{10-12}$ These two multicenter, double-masked, placebo-controlled, randomized clinical trials are the basis for the current recommendations for the use of PDT.

\section{TAP Study}

In the Treatment of Age-related Macular Degeneration with Photodynamic Therapy (TAP) Study Group, patients were included if they had a subfoveal CNV secondary to AMD with some classic component on FA, a greatest linear lesion dimension of $<5,400 \mathrm{~mm}$, and best-corrected visual acuity (BCVA) between 20/40 and 20/200 Snellen equivalents. Patients were treated with either verteporfin or placebo and followed up at three-month intervals over two years. Re-treatment was performed if the investigator determined persistent or recurrent leakage from CNV on FA. A total of 609 patients were enrolled in the study in 22 centers in Europe and the US. At the month-24 examination, the visual stabilization (loss $\leq 15$ letters) was greater in verteporfin-treated eyes (53\%) compared with the placebo-treated patients $(38 \% ; p<0.001)$. In subgroup analyses for predominantly classic lesions at baseline, $59 \%$ of verteporfin-treated patients compared with $31 \%$ of placebo-treated patients lost $<15$ letters at the month-24 examination $(p<0.001)$. For minimally classic lesions at baseline, no statistically significant differences in VA were noted. In addition to the primary outcome measure of VA, FA findings and contrast sensitivity were better in the verteporfintreated group at one and two years in all subgroups-including minimally classic lesions-than in the placebo-treated patients.

\section{Verteporfin in Photodynamic Therapy Study}

The Verteporfin in Photodynamic Therapy Study (VIP) is a multicenter, double-masked, placebo-controlled, randomized trial with two treatment arms-CNV secondary to pathological myopia and occult CNV secondary to AMD. In the group of pathological myopia (VIP PM) after a follow-up of 24 months, a loss of at least eight letters $(p=0.11)$ or more was found in $36 \%$ of the treatment group compared with $51 \%$ of the placebo group. The change in VA between baseline and the month-24 examination was in favor of cases assigned to verteporfin ( $p=0.05$ ). This included an improvement at 24 months by at least five letters in $40 \%$ of the PDT group versus $13 \%$ of the placebo-treated cases, and improvement by at least 15 letters (equivalent to at least three lines) in 12 versus $0 \%$ of the placebo-treated cases. In the second arm, the VIP AMD study included occult without classic CNV in AMD eyes." Although there was no significant difference between the treated group and the placebo group at 12-month follow-up, a minimal but significant difference in the mean VA could be pointed out at the month-24 control. At this time, visual stabilization was greater in verteporfin-treated eyes (45\%) compared with the placebo-treated patients (32\%; $p=0.032$ ).

\section{Rationale for Combination Therapy}

The use of combination therapy of intravitreal steroids or anti-vascular endothelial growth factor (VEGF) drugs with the existing PDT with verteporfin has been proposed as having the potential to enhance results synergistically, and perhaps decrease the need for re-treatment. ${ }^{24}$

\section{Photodynamic Therapy and Intravitreal Triamcinolone}

After PDT there is collateral damage to the adjacent choriocapillaris and RPE and an increase in edema and local VEGF production. ${ }^{25-26}$ since triamcinolone decreases inflammation and suppresses secretion of VEGF and stromal-derived factor 1 (SDF-1), it has been suggested that simultaneous administration of verteporfin PDT with intravitreal triamcinolone may have a synergistic effect. ${ }^{27}$ Although triamcinolone improved the efficacy of PDT with verteporfin, it also led to increases in steroid-induced glaucoma and cataract. With past results in mind, Dr Albert Augustin designed a study examining the use of triple therapy-PDT with verteporfin, bevacizumab, and the steroid dexamethasone. ${ }^{28}$ In the 28 -week 
study of 59 eyes of 59 patients, all of whom received triple therapy, VA improved in most patients. Only one cycle of treatment was required, supplemented occasionally by intravitreal injections of bevacizumab. Such an approach would require more extensive study, but it offers the possibility of lower cost and less frequent treatment. Spaide and co-workers reported a mean visual improvement compared with baseline in treatment-naïve patients, and stabilization in patients having previous PDT. ${ }^{29}$ Patients needed a mean of 1.2 treatments in the first year. Augustin and Schmidt-Erfurth treated 184 patients with verteporfin PDT and $25 \mathrm{mg}$ of intravitreal triamcinolone acetonide. On average, patients had improvements in vision of 1.22 Snellen lines and required an average of 1.21 treatments. ${ }^{30}$

\section{Photodynamic Therapy and Anti-vascular Endothelial Growth Factor Drugs}

The success of anti-VEGF agents has led to a number of potential new treatment strategies. Using a combination approach with anti-VEGF may even improve on the outcomes seen with monotherapy, offering patients better VA and/or a better angiographic result. Accompanying the reduction in number of treatments is a reduced risk of adverse events, both systemic and ocular. Potential systemic risks of long-term pan-VEGF blockade include thromboembolic events, bleeding events, and possible blockade of neuroprotection, with possible implications for stroke recovery, Alzheimer's disease, and Parkinson's disease. ${ }^{31-35}$ Hypothetical ocular risks include damage to the retinal pigment epithelium (RPE) and choriocapillaris with exacerbation of RPE geographical atrophy, and risk of neuronal damage with progressive retinal thinning.

Previous combination studies have studied ranibizumab plus PDT (FOCUS), ranibizumab plus same-day PDT (PROTECT), and pegaptanip plus PDT (VISION). Ongoing combination therapy studies include verteporfin intravitreal triamcinolone acetonide (VERITAS), evaluation of efficacy and safety in maintaining visual acuity with sequential treatment of neovascular AMD (LEVEL), ranibizumab plus PDT versus monthly ranibizumab (DENALI), and the latter's European companion study, MONT BLANC.

\section{Conclusion}

While the vision results with ranibizumab treatment for AMD from anti-VEGF antibody ranibizumab in the treatment of neovascular AMD (ANCHOR) and minimally classic/occult trial of the anti-VEGF antibody ranibizumab in the treatment of neovascular AMD (MARINA) are impressive, combination therapies with PDT may allow us to maintain or improve efficacy while reducing both systemic and ocular risk.
1. The Eye Diseases Prevalence Research Group, Prevalence of age-related macular degeneration in the United States, Arch Ophthalmol, 2004;122:564-72.

2. Klein $R$, Klein $B E$, Linton $K L$, Prevalence of age-related maculopathy. The Beaver Dam Eye Study, Ophthalmology, 1992;99:933-43.

3. Current Population Reports, Population projections of the Unites States by age, sex, race, and Hispanic origin: 1995-2050, Series P25-1130, Washington: United States Bureau of the Census, 1996. Available at: www.census.gov/prod/1/pop/p251130/ p251130.pdf

4. Fine $\mathrm{HF}$, Spaide RF, Exudative age-related macular degeneration: causes and treatment. Release date: 29 September 2006. Available at: http://www.medscape.com/viewprogram/6050_pnt

5. Mennel S, Barbazetto I, Meyer $\mathrm{CH}$, Ocular photodynamic therapy—standard applications and new indications (part 1). Review of the literature and personal experience, Ophthalmologica, 2007;221(4):216-26.

6. Green WR, Key SN 3rd, Senile macular degeneration: a histopathologic study: 1977, Retina, 2005;25(Suppl.5): 180-250.

7. Wing GL, Blanchard GC, Weiter JJ, The topography and age relationship of lipofuscin concentration in the retinal pigment epithelium, Invest Ophthalmol Vis Sci, 1978;17:601-7.

8. Sunness JS, Gonzalez-Baron J, Bressler NM, et al., The development of choroidal neovascularization in eyes with the geographic atrophy form of age-related macular degeneration, Ophthalmology, 1999;106:910-19.

9. Sunness JS, Rubin GS, Applegate CA, et al., Visual function abnormalities and prognosis in eyes with age-related geographic atrophy of the macula and good visual acuity, Ophtha/mology, 1997; 104:1677-91.

10. Treatment of Age-Related Macular Degeneration with Photodynamic Therapy (TAP) Study Group: Photodynamic therapy of subfoveal choroidal neovascularization in age related macular degeneration with verteporfin: one-year result of 2 randomized clinical trials - TAP report, Arch Ophthalmol, 1999;117: 1329-45.

11. Verteporfin in Photodynamic Therapy Study Group: Verteporfin therapy of subfoveal choroidal neovascularization in age-related macular degeneration: two-year result of a randomized clinical trial including lesions with occult with no classic choroidal neovascularization - verteporfin in photodynamic therapy report 2, Am J Ophthalmol, 2001;131:541-60.
12. Blinder KJ, Blumenkranz MS, Bressler NM, et al., Verteporfin therapy of subfoveal choroidal neovascularization in pathologic myopia: 2-year results of a randomized clinical trial - VIP report No 3, Ophthalmology, 2003;110:667-73.

13. Porrini $G$, Giovannini $A$, Amato $G$, et al., Photodynamic therapy of circumscribed choroidal hemangioma, Ophthalmology, 2003;110: 674-80.

14. Muller-Velten $R$, Michels $S$, Schmidt-Erfurth $U$, Laqua $H$, Photodynamic therapy: extended indication, Ophtha/mology, 2003;100:384-90.

15. Karacorlu M, Karacorlu S, Ozdemir H, Mat C, Photodynamic therapy with verteporfin for choroidal neovascularization in patients with angioid streaks, Am J Ophthalmol, 2002;134: 360-66.

16. Karacorlu M, Karacorlu S, Ozdemir H, Photodynamic therapy with delayed light application for the treatment of bilateral subfoveal choroidal neovascularization in age-related macular degeneration, Jpn J Ophthalmol, 2003;47(6):595-8.

17. Karacorlu M, Karacorlu S, Ozdemir H, Photodynamic therapy in patients with idiopathic choroidal neovascularization, Jpn J Ophthalmol, 2004;48(4):422-4.

18. Ozdemir H, Karacorlu SA, Karacorlu M, Early optical coherence tomography changes after photodynamic therapy in patients with age-related macular degeneration, Am J Ophthalmol, 2006;141(3):574-6.

19. Karacorlu SA, Ozdemir H, Senturk F, Karacorlu M, Optical coherence tomography after photodynamic therapy for patients with pathologic myopia, Retina, 2006;26(7):752-6.

20. Schmidt-Erfurth U, Hasan T, Gragoudas E, Vascular targeting in photodynamic occlusion of subretinal vessels, Ophtha/mology, 1994:101:1953-61.

21. Noske UM, Schmidt-Erfurth U, Meyer C, Diddens H, Lipid metabolism in retinal pigment epithelium: possible significance of lipoprotein receptors, Ophthalmologe, 1998;95:814-19.

22. Schmidt-Erfurth U, Hasan T, Schomacker K, In vivo uptake of liposomal benzoporphyrin derivative and photothrombosis in experimental corneal neovascularization, Laser Surg Med, 1995;17:178-88.

23. Kramer M, Miller JW, Michaud N, Liposomal benzoporphyrin derivate verteporfin photodynamic therapy: selective treatment of choroidal neovascularization in monkeys, Ophthalmology, 1996:103:427-38.

24. Spaide RF, Rationale for combination therapies for choroidal neovascularization, Am J Ophthalmol, 2006;141:149-56.
25. Rogers AH, Martidis A, Greenberg PB, Puliafito CA, Optical coherence tomography findings following photodynamic therapy of choroidal neovascularization, Am J Ophthalmol, 2002:134: $566-76$.

26. Schmidt-Erfurth U, Schlotzer-Schrehard U, Cursiefen C, et al., Influence of photodynamic therapy on expression of vascular endothelial growth factor (VEGF), VEGF receptor 3, and pigment epithelium-derived factor, Invest Ophthalmol Vis Sci, 2003;44:4473-80.

27. Brooks HL Jr, Caballero S Jr, Newell CK, et al., Vitreous levels of vascular endothelial growth factor and stromal-derived factor 1 in patients with diabetic retinopathy and cystoid macular edema before and after intraocular injection of triamcinolone, Arch Ophthalmol, 2004;122:1801-7.

28. Augustin AJ, Puls S, Offermann I, Triple therapy for choroidal neovascularization due to age-related macular degeneration: verteporfin PDT, bevacizumab, and dexamethasone, Retina, 2007;27(2):133-40.

29. Spaide RF, Sorenson J, Maranan L, Photodynamic therapy with verteporfin combined with intravitreal injection of triamcinolone acetonide for choroidal neovascularization, Ophtha/mology, 2005:112:301-4.

30. Augustin AJ, Schmidt-Erfurth U, Verteporfin therapy combined with intravitreal triamcinolone in all types of choroida neovascularization due to age-related macular degeneration, Ophthalmology, 2006;113:14-22.

31. Brown DM, Kaiser PK, Michels M, for the ANCHOR Study Group. Ranibizumab versus verteporfin for neovascular age-related macular degeneration, N Engl J Med, 2006;355:1432-44.

32. Rosenfeld PJ, Brown DM, Heier JS, MARINA Study Group. Ranibizumab for neovascular age-related macular degeneration, N Engl J Med, 2006;355:1419-31.

33. Zhu W, Mao Y, Zao Y, et al., Transplantation of vascular endothelial growth factor-transfected neuronal stem cells into the rat brain provides neuroprotection after transient focal ischemia, Neurosurgery, 2005;57:325-33.

34. Solerte $S B$, Ferrari E, Cuzzoni G, Decreased release of the angiogenic peptide vascular endothelial growth factor in Alzheimer's disease, Dement Geriatr Cogn Disorder, 2005;19: $1-10$.

35. Yasuhara T, Shingo T, Kobayashi K, et al., Neuroprotective effects of vascular endothelial growth factor (VEGF) upon dopaminergic neurons in a rat model of Parkinson's disease, Eur J Neurosci, 2004;19(6):1494-1504. 